\title{
Imaging-guided fine tuning of CAR synapse dynamics and T cell triggering
}

Casey Beppler ${ }^{1}$, John Eichorst ${ }^{2}$, Kyle Marchuk ${ }^{2}$, En Cai ${ }^{1}$, Carlos A. Castellanos ${ }^{3}$, Venkataraman Sriram ${ }^{4}$, Kole T. Roybal ${ }^{5,6,7,8}$ and Matthew F. Krummel ${ }^{1^{*}}$

${ }^{1}$ Department of Pathology and ImmunoX, University of California, San Francisco, San Francisco, CA 94143, USA; ${ }^{2}$ Biological Imaging Development CoLab, University of California, San Francisco, San Francisco, CA 94143, USA; ${ }^{3}$ Department of Microbiology and Immunology, Sandler Asthma Basic Research Center, University of California, San Francisco, San Francisco, CA 94143, USA; " Foundery Innovations, San Francisco, CA 94107, USA; ${ }^{5}$ Department of Microbiology and Immunology, University of California, San Francisco, San Francisco, CA 94143, USA; ${ }^{6}$ Parker Institute for Cancer Immunotherapy; ${ }^{7}$ Chan Zuckerberg Biohub; ${ }^{8}$ Helen Diller Comprehensive Cancer Center

${ }^{*}$ Corresponding author
Abstract
T cells typically recognize their ligands using microvilli to scan for cognate antigen and scaffold
T cell receptors (TCRs) during antigen recognition. Conventional chimeric antigen receptors
(CARs) are often built using single-chain variable fragments (scFvs) with far greater affinity than that of natural TCRs. The implications of this for T cell function are not well understood. Using high-resolution microscopy, we studied the membrane dynamics in cells bearing anti-human epidermal growth factor receptor 2 (HER2) CARs and found these hyper-stabilized microvillar contacts relative to TCRs. While these CARs also impaired synapse resolution, a monomerized CAR with a lower affinity scFv rescued synapse dynamics and improved antigen-dose discrimination. The dimeric low-affinity CAR improved synapse dynamics and minimized early indicators of exhaustion, while maintaining or improving effector functions. This work highlights the importance of designing CAR binding dynamics that more closely resemble natural TCR antigen sensing to optimize $\mathrm{T}$ cell quality and function. 


\section{Introduction}

The development of CAR T cells revolutionized the field of cancer immunotherapy, proving highly successful in the treatment of B cell malignancies ${ }^{1-4}$. Typical CAR designs incorporate an antigen-binding scFv, along with intracellular $\mathrm{CD} 3 \zeta$ and co-stimulatory domains - commonly from either 4-1BB or CD28. Signaling events initiated by scFv binding and subsequent phosphorylation of intracellular components at the immune synapse lead to the acquisition of CAR T cell effector functions.

Natural TCR signaling at the prototypical immune synapse involves the local accumulation of signaling components in microclusters, which can further segregate at later stages into canonical central supramolecular activation clusters (cSMAC) $)^{5-7}$. Microclusters are thought to be conducive to signal initiation and amplification - allowing segregation of phosphatases away from signaling domains. The CSMAC - where active kinases are rare - is a site where TCR signaling is thought to end ${ }^{5}$. More recent work has revealed the three-dimensional topography underlying synapse formation, involving dynamic microvillar search during antigen scanning, and stabilization following cognate antigen binding ${ }^{8}$. Whether and how CARs engage this native cell biology are largely unknown.

For some CAR T cell therapies, it is critical to discriminate tumor cells from healthy cells bearing the identical ligands. Given the lack of tumor-specific antigens, one strategy has been to target tumor-associated antigens, such as HER2, which are expressed at relatively higher levels in tumor than healthy tissue. In these cases, the on-target recognition of off-tumor antigen present at low levels can lead to serious toxicities ${ }^{9}$. Several strategies have been proposed in attempts to circumvent this problem ${ }^{10-17}$. However, little is known about the cell biology and mechanics underlying a CAR-based immune synapse. 
CAR T cell strategies at present do not produce ideal outcomes, particularly in solid tumors. Problems include lack of CAR T cell infiltration and persistence, incomplete tumor clearance and escape variants, as well as a lack of actionable tumor antigens as alluded to above $\mathrm{e}^{18,19}$. Recent work also suggests that gross CAR aggregation, caused by unintended interactions in scFv framework regions, results in over-triggering of T cells via tonic signaling and exhaustion, which can be ameliorated using 4-1BB co-stimulation ${ }^{20}$. The parameters affecting exhaustion in 4-1BB CARs that do not so distinctly aggregate, are not well understood.

To shed light on how CARs integrate the existing cell biology underlying T cell triggering, we combined high-resolution imaging of CAR T cell synapse dynamics with an analysis of T cell function. We studied a model CAR targeting HER2 in conjunction with targets bearing different levels of ligand and addressed how this CAR localizes using lattice light-sheet imaging (LLS) and synaptic contact mapping (SCM) total internal reflection fluorescence (TIRF) microscopy. We then used a series of CARs with variable binding dynamics to measure how these affect synapse stabilization and resolution. In doing so, we found that CAR interactions with hyperstable binding dynamics—as a result of affinity, dimerization, or antigen density_impair synapse resolution and promote exhaustion. 


\section{Results}

We expressed anti-HER2 CARs in primary human CD8+ T cells by lentiviral transduction and first sought to compare how they distribute on the T cell surface, compared to conventional TCR. To do this, we first fixed CAR T cells, stained them with antibodies that bound to TCR and CAR, and imaged them by LLS. Visually, this revealed that CAR was distributed across the surface of the cell with patches of increased fluorescence intensity, similar to that of TCR although not necessarily overlapping in position (Fig. 1a, Extended Data Fig. 1a and Supplemental Video 1). To quantify the degree of clustering, the fluorescence intensity at the membrane was mapped to radial intensity profiles (Fig. 1b and Extended Data Fig. 1b). Taking a moving average with a bin size of 10 pixels, patches of increased local fluorescence were identified for TCRs, as expected ${ }^{21}$, but not for the CD45 negative control (Extended Data Fig. 1c). Using this method, CARs were found to form patches, similarly to TCRs (Fig. 1c-d). Thus, on an isolated T cell, anti-HER2 CARs were found to distribute in a pattern similar to that of the natural TCR, although not necessarily overlapping.

Next, in order to determine the location of CARs following interaction with a target cell, live antiHER2 CAR T cells were added to coverslips loaded with HER2+ SKBR3 cells and imaged by LLS at intervals of 4.7 secs. This live cell imaging revealed dynamic movements of CAR patches and their accumulation over time at microvillar close contacts, (Fig. 1e and

Supplemental Video 2) similar to what we have observed for $\mathrm{TCR}^{8}$. To further study the localization of CARs to microvillar close contacts, T cells expressing an mEmerald-labeled CAR were analyzed on HER2-loaded lipid bilayers using SCM TIRF microscopy. This method allows for visualization of both the receptor by TIRF, in a green fluorescent channel, and the location of microvillar close contacts, as seen by holes in fluorescent quantum dot (QD605) signal (Extended Data Fig. 1d). Microclusters of CAR-mEmerald fluorescence were found to localize to areas of microvillar close contacts, as seen by a line scan revealing segregation between the 
two signals (Fig. 1f-g). To determine whether this localization was dependent on interaction with a HER2+ surface, anti-HER2 CARs were expressed in OT-I mouse T cells and allowed to interact with bilayers loaded with peptide-major histocompatibility complex (pMHC):SL8 (SIINFEKL). In these HER2-negative synapses mediated by TCR-pMHC binding, CARs were found to localize across the synapse, without enrichment in microvilli (Extended Data Fig. 1e-f). Thus, we found that the recruitment of CAR microclusters to the microvillar close contacts relied upon the presence of CAR antigen on the interacting surface.

We next sought to understand how microvillar close contacts, hallmarks of TCR-induced activation ${ }^{8,22}$, were modulated by the affinity of the CAR for its ligands. To do this, we compared the wild-type high-affinity scFv (4D5) with a mutated scFv (mut4D5, Fig. 2a and Supplementary Table 1) of $\sim 70$-fold reduced affinity. For our study, we also used two breast cancer cell lines that differed significantly in their HER2 levels: the MCF7 luminal breast cancer cell line does not over-express HER2, and thus serves as a counterpart to healthy tissue level expression; and the SKBR3 cell line endogenously over-expresses HER2, and thus mirrors HER2-positive breast cancers ${ }^{23}$ (Extended Data Fig. 2a). T cells bearing high-affinity CARs formed stable cell:cell interactions with high-HER2 expressing SKBR3s, as did T cells bearing low-affinity CARs interacting with low-HER2 expressing MCF7s, as seen by LLS (Extended Data Fig. 2b). We then analyzed microvillar close contacts by SCM at high temporal resolution using graded doses of ligands, with $62.5 \mathrm{ng}$ per $0.7 \mathrm{~cm}^{2}$ well representing low-level expression on the order of MCF7s (Fig. 2b and Extended Data Fig. 2c). In the examples in Fig. 2c and Supplemental Video 3, individual microvillar close contacts mediated by low-HER2 bilayers remained stable in the case of a high-affinity CAR contact, while the low-affinity CAR close contact dispersed within seconds. Quantifying this, individual microvillar close contacts were tracked and their lifetime was measured using automated scripts that identify regions of QD605exclusion, revealing that the persistence time of close contacts increases with CAR affinity at 
low antigen densities, but was indistinguishable at high antigen density (Fig. 2d). Notably, at high HER2 density, the persistence of low-affinity CAR close contacts reached $\sim 17$ secs on average, a value that was achieved by high-affinity CAR even at $100 x$ lower antigen densities lower even than the low-HER2 MCF7 standard. Notably, both low (62.5 ng/well) and high (625 ng/well) antigen densities led to calcium flux as detected by Fura-2 ratiometric imaging (Extended Data Fig. 2d-f and Supplemental Video 4). At high density of antigen, we also observed a decrease in the magnitude of the maximal ratio achieved, specifically for the highaffinity CAR interactions. In summary, this suggested an altered dose response in the highaffinity setting.

Given the 17 secs persistence time was greater than the $\sim 11$ secs observed in a previous study of TCR-mediated microvillar stabilization ${ }^{8}$, we sought to directly compare CAR- and TCRmediated persistence times. We thus expressed the low-affinity CAR in OT-I mouse T cells and compared interactions with SL8:pMHC-, low HER2-, and high HER2-loaded bilayers.

Interactions of low-affinity CAR with low-HER2 bilayers yielded similar persistence times to that of the natural TCR (Fig 2e). Close contact persistence times on high HER2 were found to be significantly greater than that of TCR-mediated stabilization. Thus, CARs on high HER2-loaded bilayers yielded hyper-stable microvillar dynamics, i.e. above the natural stabilization of TCRs. Not all microvillar close contacts were occupied by CAR in synapses with cognate antigen, as is the case for TCR (Extended Data Fig. 2g). Given that low-affinity CARs behaved similarly to TCRs at low HER2 densities, we hypothesized that we could further engineer the low-affinity CAR to yield TCR-like persistence times in high antigen-density conditions by minimizing CAR avidity contributions. Thus, we created a monomeric CAR by incorporating two cysteine to serine point mutations in the CD8a hinge to prevent formation of the disulfide bridge ${ }^{24}$ (Extended Data Fig. 2h). Average CAR fluorescence intensity of close contacts was not significantly different between monomers and dimers (Extended Data Fig. 2i). Comparing the 
persistence time of monomers to dimers on high HER2-loaded bilayers revealed that only the low-affinity monomers produced microvillar persistence similar to that of the TCR (Fig. $\mathbf{2 f}$ ). Average close contact CAR fluorescence intensity did not appear to correlate with persistence times under the conditions tested (Extended Data Fig. 2j).

TCR-mediated synapses formed on antigen-loaded lipid bilayers result in TCR microcluster formation and radial motion of microclusters toward the center into what eventually becomes the $\mathrm{CSMAC}^{7}$. Although alternative synapse architectures have been revealed for CAR-mediated synapses $^{25}$, we sought to determine whether binding dynamics were a key regulator of this centralization. Endpoint measurement of the distance across T cell microclusters following TIRF imaging of synapses revealed that centralization of dimeric CAR microclusters only occurred in conditions of low affinity and low antigen density (Fig. 3a-b). On the high HER2-loaded bilayer, the low-affinity CAR monomer had the tightest centralization of microclusters (Fig $\mathbf{3 b}$ ). Thus, supra-physiological binding dynamics, affected by affinity, dimerization, and/or antigen density, were found to impair typical synapse resolution, i.e. with centrally accumulated antigen receptor. In order to determine whether this phenotype was the result of a lack of mobility of microclusters, as opposed to direct accumulation of CARs in the center vs. periphery, we tracked the microclusters over time (Extended Data Fig. 3a and Supplemental Videos 5-10). On high-HER2 bilayers, the low-affinity CAR monomer yielded mobile microclusters, whereas both CAR dimers showed impaired mobility by examination of flower plot, displacement, and speed (Fig. 3c-d and Extended Data Fig. 3b-c). The mobility of low-affinity CAR dimer microclusters was restored on low HER2-loaded bilayers, while the high-affinity CAR dimer mobility did not change compared to the high HER2 density condition (Extended Data Fig. 3bc). Analysis of the direction of microcluster movement revealed no significant differences (Extended Data Fig. 3d). Therefore, we found that a monomerized low-affinity CAR supported 
the formation of synapse architectures and dynamics characteristic of TCRs on antigen-loaded lipid bilayers, whereas high-affinity dimerized CARs did not.

In order to determine downstream effects of the hyper-stable or more physiological receptors, we incubated CAR T cells with low-HER2 expressing MCF7s or high-HER2 expressing SKBR3s and measured a series of outputs to compare activation, proliferation, and effector function.

(Fig. 4a). As measured by CD69 staining at 18 hours following stimulation, similarly robust activation was observed for all CAR interactions with low- or high-HER2 targets, (Fig. 4b, Extended Data Fig. 4a). Proliferation as measured by VPD dilution after 96 hours varied significantly and the greatest proliferation was observed using low-affinity dimeric CAR (Fig. 4cd). Cells stained at 96 hours post-stimulation showed equivalent levels of Ki67 staining (Fig. 4e).

Intracellular staining for IFN-y at 18 hours post-stimulation also revealed increased production of this cytokine in cells bearing low-affinity CAR dimers relative to high-affinity ones (Fig. 4f). We observed that, specifically for human T cell co-cultures, less IFN-y was produced by dimeric CAR T cells when incubated with high-HER2 SKBR3s than with low-HER2 MCF7s. In this regard, while the low-affinity monomeric CAR displayed a decreased magnitude of response, it restored the expected dose-dependent response pattern with higher antigen density yielding higher IFN-Y.

We next sought to address the effects of altered kinetics using an in-vivo tumor model with immunocompetent hosts, to more closely match physiological settings. MC38 tumor cells were thus transduced with truncated human HER2, lacking intracellular signaling domains, and sorted into MC38-HER2-low and MC38-HER2-high tumor cell lines. In-vitro co-cultures of high and low-affinity dimeric receptors revealed that the low-affinity CAR T cells performed similarly to the high-affinity CAR T cells across antigen density levels, as measured by IFN- $\gamma$ intracellular staining at 18 hours following co-culture. In mouse T cells, dose responses followed the 
expected pattern, i.e. IFN-y production increased with antigen dose (Extended Data Fig. 5a). Tumor growth experiments using this system also revealed no significant difference in tumor control between the high and low-affinity CARs (Extended Data Fig. 5b-d). Longer-lived memory and low exhaustion phenotypes have been identified as key indicators of CAR T cell quality ${ }^{18,26}$. There were no significant differences between $T$ cells when analyzed at the pretransfer time point for central memory:effector ratio, TOX, or PD1 (Extended Data Fig. 6a). For MC38-HER2-low tumors that were large enough for harvest, we noted no significant differences in tumor-infiltrating untransduced or CAR T cells for central memory:effector ratio, TOX, or PD1 (Extended Data Fig. 6b-d, 7a-b). Together this suggests little benefit for the high-affinity receptor.

Given that in most cases it was not feasible to harvest and characterize tumor-infiltrating CAR T cells (due to negligible tumor size), we designed co-culture experiments to analyze T cell phenotypes under hypoxia, which more closely mimics the solid tumor environment and has been shown to contribute to exhaustion ${ }^{27}$. Following 6 days of incubating $T$ cells with ligandbearing cells in hypoxia, TOX was found to be significantly increased in T cells bearing highaffinity CARs and cultured with high antigen tumor cells, indicating that hyper-stable binding dynamics yield T cell exhaustion under hypoxic conditions (Fig. 4g, Extended Data Fig. 4b). CAR T cells that were both TOX high and PD1+ were also significantly increased in hypoxic cultures of high-affinity CAR T cells with high HER2 (Extended Data Fig. 8a). Other exhaustion markers - PD1, LAG3 and CD39 - were also increased in hypoxic cultures for high and lowaffinity CAR T cell co-cultures with high HER2 (Fig. 4g and Extended Data Fig. 8b). 


\section{Discussion}

While impressive results have come from CAR therapies targeting certain B cell malignancies ${ }^{1-}$ ${ }^{4}$, efforts to translate that success to solid tumors have revealed deficiencies in current CAR T cell biology. Although specific targeting of tumor antigens is one of these challenges, not much is known about whether and how CARs engage the natural T cell biology underlying antigen scanning, or how variations in binding affect synapse dynamics and CAR triggering.

Finding the optimal balance between robust effector function and exhaustion is one of the current challenges in the field. Our work suggests that binding dynamics may be a key feature of CAR design which could modulate this axis. The dimeric lower affinity CAR tested here reduced TOX expression, a known regulator of commitment to exhaustion ${ }^{28,29}$, under hypoxia with continuous stimulation. Exhaustion of CAR T cells caused by substantial tonic signaling has been identified as a barrier to successful outcomes and various methods have been designed to minimize these effects ${ }^{20,30-33}$. Compared to those studies, we did not observe substantial exhaustion generated in the absence of antigen stimulation in vitro, which is likely due to minimal multimerization of the CARs tested here - LLS imaging of CARs shows patches of locally high intensity similar to that of the natural TCR - but may also be aided by our use of 41BB co-stimulation which is thought to be less prone to promoting exhaustion ${ }^{20}$.

Desirable outcomes have been ascribed previously to the use of lower affinity CARs - for example, a very low affinity scFv $\left(4 \mathrm{D} 5-3, \mathrm{~K}_{\mathrm{D}}=3,910 \mathrm{nM}\right)$ CAR improved antigen density discrimination ${ }^{12}$. This is an important quality given the desire to minimize toxicities resulting from on-target, off-tumor binding. Of note, the lower affinity scFv tested here $\left(K_{D}=3.6 n M\right)$ is of much greater affinity than 4D5-3, and the dimeric CAR using this scFv still demonstrates poor antigen density discrimination. While the monomer improved discrimination, this comes at the cost of reduced effector responses. Future work will be needed to determine whether boosting the 
intracellular signal downstream of monomeric binding dynamics would yield both precise antigen density discrimination and robust signaling.

Previous work by Davenport et al. established the existence of altered CAR T cell synapse structures defined by a multifocal distribution of Lck microclusters, which were associated with more rapid response times relative to those mediated by native $\mathrm{TCR}^{25}$. Our work expands on this by showing that an anti-HER2 CAR drives microclusters to form at microvillar contacts but generates hyper-stability of the underlying protrusion. Additionally, by variation of affinity and multimerization status of a CAR we show that binding dynamics, and possibly the intracellular multimerization state, are a key regulator of CAR microcluster mobility and synapse structure, with high-affinity/high-antigen interactions leading to impaired synapse resolution. Highaffinity/high-antigen interactions are also associated with reductions in T cell quality - scFvs with $\sim 70$-fold reduced $K_{D}$ maintained or enhanced effector function and improved resistance to exhaustion.

Reduced activation has been described for TCR-pMHC interactions with supraphysiological binding dynamics ${ }^{34-37}$, which may be in part due to increased SHP-1 activity ${ }^{38}$. Less commonly described are the effects of high affinity and antigen density on CAR responsiveness ${ }^{17,39,40}$. Here we find these to be superoptimal in experiments measuring proliferation and IFN- $\mathrm{Y}$ in human CAR T cells. However, we have studied only two scFvs, both of which have higher affinities for their ligands compared to typical TCRs. Future work will be needed to determine if the same mechanisms are at play in enforcing upper limits on CAR and TCR binding dynamics. In general, we expect the optimal scFv binding affinity to be greater than that of a TCR, based on the demonstration that CAR intracellular signaling domains are relatively inefficient ${ }^{39}$ and that in some cases, higher affinity CARs can lead to increased potency ${ }^{41}$. Optimal binding dynamics will likely vary based on other aspects of CAR design and choice of signaling domains, but our 
evidence indicates that this optimization step is a critical component of high-quality $\mathrm{T}$ cell engineering.

Here we show that although CARs assemble on the membrane as patches of receptors much like TCR, the distinctness of their synthetically engineered transmembrane domain - taken from CD8 - and intracellular domains appear to result in an initial propensity to segregate as patches independently of TCRs, i.e. not necessarily overlapping in position. We hypothesize that this results from differential miscibility and recruitment of membrane lipids related to combinations of transmembrane and cytoplasmic domains. Optimization of this may provide an additional benefit to engineered receptors by matching the recruitment of adapters to those of the native TCR, but goes beyond the focus of this paper.

To our knowledge, this work is the first to study engagement of microvillar scanning mechanisms by CARs, and also to assess variations in synapse architecture downstream of CARs targeting the same antigen but with different binding dynamics. Distinct synapse architectures have been reported as being associated with different cell types and signaling strength, as well as variable downstream outcomes ${ }^{42}$. One example is that of the multifocal synapse that forms during thymic negative selection ${ }^{43}$. In TCR synapses, slower receptor unbinding kinetics have been associated with reduced actin velocity ${ }^{44}$. Whether similar mechanisms are at play in multifocal CAR synapses is unknown. While this work demonstrates that CAR binding dynamics alter synapse architecture and T cell quality, this work does not show whether differences in synapse resolution are causal to effects on effector function and exhaustion. Future work is warranted to determine whether altering synapse dynamics to more closely match the natural cell biology would improve T cell quality. For future CAR engineering, synapse dynamics could be an early predictor of ultimate T cell quality. 


\section{Author Contributions}

C.B. designed, conducted, and analyzed most experiments, and drafted the manuscript. J.E. and K.M. authored unique MatLab codes and discussed data. K.M. also assisted with LLS imaging acquisition. E.C. discussed project design. C.A.C. performed mouse irradiations and discussed data. V.S. designed affinity measurement experiments. K.T.R. contributed to the formulation of the project, designed experiments, provided reagents for experiments involving human T cells, and interpreted data. M.F.K. contributed to the formulation of the project, designed experiments, interpreted data, and developed the manuscript. All authors contributed manuscript revisions.

\section{Competing Interests}

K.T.R. is a cofounder, consultant, SAB member, and stockholder of Arsenal Biosciences. K.T.R. is on the SAB of Ziopharm Oncology and an Advisor to Venrock.

\section{Data and Materials Availability}

The datasets and unique materials generated during the current study are available from the corresponding author on reasonable request.

\section{Code Availability}

Unique analysis codes have been made available and can be accessed via the GitHub links provided in the relevant methods sections. 


\section{References}

1. Kalos, M. et al. T cells with chimeric antigen receptors have potent antitumor effects and can establish memory in patients with advanced leukemia. Sci. Transl. Med. 3, 1-13 (2011).

2. Brentjens, R. J. et al. CD19-targeted T cells rapidly induce molecular remissions in adults with chemotherapy-refractory acute lymphoblastic leukemia. Sci. Transl. Med. 5, (2013).

3. Maude, S. L. et al. Chimeric Antigen Receptor T Cells for Sustained Remissions in Leukemia. New England Journal of Medicine vol. 371 1507-1517 (2014).

4. Turtle, C. J. et al. CD19 CAR-T cells of defined CD4+:CD8+ composition in adult B cell ALL patients. J. Clin. Invest. 126, 2123-2138 (2016).

5. Campi, G., Varma, R. \& Dustin, M. L. Actin and agonist MHC-peptide complex-dependent T cell receptor microclusters as scaffolds for signaling. J. Exp. Med. 202, 1031-1036 (2005).

6. Grakoui, A. et al. The Immunological Synapse: A Molecular Machine Controlling T Cell Activation. 221, (1999).

7. Monks, C. R., Freiberg, B. A., Kupfer, H., Sciaky, N. \& Kupfer, A. Three-dimensional segregation of supramolecular activation clusters in T cells. Nature 395, 82-86 (1998).

8. Cai, E. et al. Visualizing dynamic microvillar search and stabilization during ligand detection by T cells. Science (80-. ). 356, (2017).

9. Morgan, R. A. et al. Case report of a serious adverse event following the administration of t cells transduced with a chimeric antigen receptor recognizing ERBB2. Mol. Ther. 18, 843-851 (2010). 
10. Srivastava, S. et al. Logic-Gated ROR1 Chimeric Antigen Receptor Expression Rescues T Cell-Mediated Toxicity to Normal Tissues and Enables Selective Tumor Targeting. Cancer Cell 35, 489-503.e8 (2019).

11. Roybal, K. T. et al. Precision Tumor Recognition by T Cells with Combinatorial AntigenSensing Circuits. Cell 164, 770-779 (2016).

12. Liu, X. et al. Affinity-Tuned ErbB2 or EGFR Chimeric Antigen Receptor T Cells Exhibit an Increased Therapeutic Index against Tumors in Mice. Cancer Res. 75, 3596-3607 (2015).

13. James, J. R. Tuning ITAM multiplicity on T cell receptors can control potency and selectivity to ligand density. Sci. Signal. 11, (2018).

14. Majzner, R. G. et al. Tuning the antigen density requirement for car T-cell activity. Cancer Discov. 10, 702-723 (2020).

15. Hernandez-Lopez, R. A. et al. T cell circuits that sense antigen density with an ultrasensitive threshold. Science (80-. ). 371, 1166 LP - 1171 (2021).

16. Caruso, H. G. et al. Tuning sensitivity of CAR to EGFR density limits recognition of normal tissue while maintaining potent antitumor activity. Cancer Res. 75, 3505-3518 (2015).

17. Park, S. et al. Micromolar affinity CAR T cells to ICAM-1 achieves rapid tumor elimination while avoiding systemic toxicity. Sci. Rep. 7, 1-15 (2017).

18. Lim, W. A. \& June, C. H. The Principles of Engineering Immune Cells to Treat Cancer. Cell 168, 724-740 (2017).

19. Majzner, R. G. \& Mackall, C. L. Tumor antigen escape from CAR T-cell therapy. Cancer Discov. 8, 1219-26 (2018). 
20. Long, A. H. et al. 4-1BB costimulation ameliorates T cell exhaustion induced by tonic signaling of chimeric antigen receptors. Nat. Med. 21, 581-590 (2015).

21. Hu, Y. S., Cang, H. \& Lillemeier, B. F. Superresolution imaging reveals nanometer- and micrometer-scale spatial distributions of T-cell receptors in lymph nodes. Proc. Natl. Acad. Sci. U. S. A. 113, 7201-7206 (2016).

22. Farrell, M. V., Webster, S., Gaus, K. \& Goyette, J. T Cell Membrane Heterogeneity Aids Antigen Recognition and T Cell Activation. Front. Cell Dev. Biol. 8, 1-9 (2020).

23. Mota, A. de L. et al. Molecular characterization of breast cancer cell lines by clinical immunohistochemical markers. Oncol. Lett. 13, 4708-4712 (2017).

24. Hennecke, S. \& Cosson, P. Role of transmembrane domains in assembly and intracellular transport of the CD8 molecule. J. Biol. Chem. 268, 26607-26612 (1993).

25. Davenport, A. J. et al. Chimeric antigen receptor T cells form nonclassical and potent immune synapses driving rapid cytotoxicity. Proc. Natl. Acad. Sci. U. S. A. 115, E2068E2076 (2018).

26. McLellan, A. D. \& Ali Hosseini Rad, S. M. Chimeric antigen receptor T cell persistence and memory cell formation. Immunol. Cell Biol. 97, 664-674 (2019).

27. Scharping, N. E. et al. Mitochondrial stress induced by continuous stimulation under hypoxia rapidly drives T cell exhaustion. Nat. Immunol. 22, 205-215 (2021).

28. Alfei, F. et al. TOX reinforces the phenotype and longevity of exhausted T cells in chronic viral infection. Nature 571, 265-269 (2019).

29. Khan, O. et al. TOX transcriptionally and epigenetically programs CD8(+) T cell exhaustion. Nature 571, 211-218 (2019). 
30. Hyrenius-Wittsten, A. et al. SynNotch CAR circuits enhance solid tumor recognition and promote persistent antitumor activity in mouse models. Sci. Transl. Med. 13, (2021).

31. Choe, J. H. et al. SynNotch-CAR T cells overcome challenges of specificity, heterogeneity, and persistence in treating glioblastoma. Sci. Transl. Med. 13, (2021).

32. Weber, E. W. et al. Transient rest restores functionality in exhausted CAR-T cells through epigenetic remodeling. Science (80-. ). 372, (2021).

33. Eyquem, J. et al. Targeting a CAR to the TRAC locus with CRISPR/Cas9 enhances tumour rejection. Nature 543, 113-117 (2017).

34. Corse, E., Gottschalk, R. A., Krogsgaard, M. \& Allison, J. P. Attenuated T cell responses to a high-potency ligand in vivo. PLoS Biol. 8, 1-12 (2010).

35. McMahan, R. H. et al. Relating TCR-peptide-MHC affinity to immunogenicity for the design of tumor vaccines. J. Clin. Invest. 116, 2543-2551 (2006).

36. Kalergis, A. H. et al. Efficient T cell activation requires an optimal dwell-time of interaction between the TCR and the pMHC complex. Nat. Immunol. 2, 229-234 (2001).

37. Schmid, D. A. et al. Evidence for a TCR Affinity Threshold Delimiting Maximal CD8 T Cell Function. J. Immunol. 184, 4936 LP - 4946 (2010).

38. Hebeisen, M. et al. SHP-1 phosphatase activity counteracts increased T cell receptor affinity. J. Clin. Invest. 123, 1044-1065 (2013).

39. Harris, D. T. et al. Comparison of T Cell Activities Mediated by Human TCRs and CARs That Use the Same Recognition Domains. J. Immunol. 200, 1088-1100 (2018).

40. Ghorashian, S. et al. Enhanced CAR T cell expansion and prolonged persistence in pediatric patients with ALL treated with a low-affinity CD19 CAR. Nat. Med. 25, 1408- 
1414 (2019).

41. Hudecek, M. et al. Receptor affinity and extracellular domain modifications affect tumor recognition by ROR1-specific chimeric antigen receptor T cells. Clin. Cancer Res. 19, 3153-3164 (2013).

42. Friedl, P., Den Boer, A. T. \& Gunzer, M. Tuning immune responses: Diversity and adaptation of the immunological synapse. Nat. Rev. Immunol. 5, 532-545 (2005).

43. Richie, L. I. et al. Imaging synapse formation during thymocyte selection: Inability of CD3ろ to form a stable central accumulation during negative selection. Immunity $16,595-606$ (2002).

44. Colin-York, H. et al. Cytoskeletal Control of Antigen-Dependent T Cell Activation. Cell Rep. 26, 3369-3379.e5 (2019).

45. Beemiller, P., Jacobelli, J. \& Krummel, M. F. Integration of the movement of signaling microclusters with cellular motility in immunological synapses. Nat. Immunol. 13, 787-795 (2012).

46. Beemiller, P., Jacobelli, J. \& Krummel, M. Imaging and Analysis of OT1 T Cell Activation on Lipid Bilayers. Protoc. Exch. (2012) doi:10.1038/protex.2012.028. 


\section{Materials and Methods}

\section{Lentiviral and retroviral CAR constructs}

All CARs were fused to C-terminal MYC tag, CD8 $\alpha$ hinge/transmembrane domain, 4-1BB costimulatory domain, $\mathrm{CD} 3 \zeta$ signaling domain, and an $\mathrm{N}$-terminal mEmerald tag. Monomeric versions of each CAR were created by using the Q5 Site Directed Mutagenesis Kit (NEB \#E0554S) yielding two cysteine to serine point mutations in the CD8 $\alpha$ hinge ${ }^{24}$ (Supplemental Table 1).

\section{Human T cell culture, lentiviral transduction, and co-incubations}

Lenti-X 293T cells (Takara Bio) were transfected with pHR SIN including cloned transgene and packaging vectors pMD2.G and pCMVdR8.91 using TransIT-Lenti Transfection Reagent (Mirus \#MIR6603). On the day of transfection, primary human CD8+ T cells were thawed into complete human T cell media: X-VIVO 15 (Lonza \# 04-418Q), 5\% Human AB serum (Valley Biomedical \#HP1022) and 10 mM neutralized N-acetyl L-Cysteine (Sigma Aldrich \#A9165-25G) supplemented with $30 \mathrm{U} / \mathrm{mL}$ recombinant human IL-2 (R\&D Systems \#202-IL), and $55 \mu \mathrm{M}$ betamercaptoethanol (Thermo \# 21985023). The following day, Human T-Activator CD3/CD28 Dynabeads (Thermo \#11161D) were added at 1:1 ratio with thawed T cells. The next day, T cell media was replaced with Lenti-X 293T viral supernatant. For lentiviral transduction of monomeric CARs, virus was concentrated by $\mathrm{PEG} / \mathrm{NaCl}$ precipitation, and stored at $-80^{\circ} \mathrm{C}$ prior to use. Viral supernatant was replaced with fresh $\mathrm{T}$ cell media the next day, and $\mathrm{T}$ cells were allowed to recover for one day prior to Dynabead removal and sort. Cells were sorted for CARmEmerald expression in the range of 1-2 logs above background. Cells were then rested and used at 10-21 days post initial stimulation. Lenti-X 293T cells were cultured in Dulbecco's modified Eagle's medium (DMEM, Gibco \#11995) with 10\% fetal bovine serum (MilliporeSigma), penicillin (50 U/ml) and streptomycin (50 $\mathrm{gg} / \mathrm{ml})$ (MP Biochemicals \#MP091670049), and $1 \mathrm{mM}$ 
sodium pyruvate (Sigma-Aldrich \#S8636). For early activation and intracellular cytokine assays, $5 \times 10^{4} \mathrm{~T}$ cells were added at a 1:1 ratio to 96 -well flat-bottom plates with MCF7 (ATCC) or SKBR3 (ATCC) cells for 18 hours. BD GolgiPlug (\#555029) was added for the final 10 hours. For proliferation assays, T cells were stained with Violet Proliferation Dye (BD \#562158) prior to plating of $2 \times 10^{4} \mathrm{~T}$ cells at 1:1 ratio with MCF7s or SKBR3s. Complete T cell media was supplemented the following day, and cells were analyzed by flow cytometry at 96 hours following plating.

\section{Flow cytometric analysis}

Zombie NIR Fixable Viability Kit (BioLegend \#423106) was used for exclusion of dead cells. Surface staining was performed with anti-mouse Fc receptor antibody (clone 2.4 G2, UCSF Hybridoma Core) or Human TruStain FcX (BioLegend \#422302) in PBS with 2\% FCS for 30 min on ice. Supplemental Table 2 lists all antibodies referenced for flow cytometry and imaging experiments. For experiments with staining of nuclear proteins, eBioscience Foxp3 / Transcription Factor Staining Buffer Set (Fisher Scientific \#00-5523-00) was used for fixation and permeabilization. For all other experiments involving intracellular staining, BD Cytofix/Cytoperm (\#554722) was used. Following fixation and permeabilization, cells were incubated with Fc block for 10 min on ice prior to addition of intracellular stain. Flow cytometry was performed on a BD Fortessa instrument, and sorting was performed on BD FACSAria or BD FACSAria Fusion instruments. FlowJo software (BD Biosciences) was used for all analyses.

\section{Mice}

Mice were housed at the University of California, San Francisco, according to Laboratory Animal Resource Center guidelines. Protocols were approved by the Institutional Animal Care and Use Committee of the University of California. C57BL/6J and B6.SJL-Ptprc ${ }^{\mathrm{a}}$ Pepc ${ }^{\mathrm{b}} /$ BoyJ (CD45.1) 
mice for tumor growth experiments were purchased from The Jackson Laboratory. Other mice were bred at the University of California, San Francisco.

\section{MC38-HER2 retroviral transduction}

Truncated HER2, without intracellular signaling domains, (NP_004439.2; amino acids 1 to 730) was cloned into pIB2 retroviral vector. Phoenix cells were transfected using FuGENE 6 Transfection Reagent (Promega \#E2691), and retroviral supernatant was collected and used immediately for transfection of MC38s on days 2 and 3 following transfection. Two days after the second transduction, cells were sorted for expression of HER2 (stained with anti-HER2A488). MC38-HER2 cells were then expanded and the retroviral transduction process was repeated. Following the second transduction, cells were then sorted into high-, medium-, and low-expression levels using MCF7 and SKBR3 cells as standards for low and high expression, respectively. MC38 cells were cultured in DMEM (Gibco \#11995) supplemented with 10\% fetal bovine serum (Benchmark), $100 \mathrm{U} / \mathrm{mL}$ penicillin, $0.1 \mathrm{mg} / \mathrm{mL}$ streptomycin, $2 \mathrm{mM} \mathrm{L-glutamine}$ (Gibco \#10378), 10 mM HEPES (Thermo \#15630106), and $55 \mu \mathrm{M}$ beta-mercaptoethanol (Thermo \# 21985023).

\section{Murine T cell culture, retroviral transduction, and functional assays}

The mouse OT-I TCR system was chosen as a comparator to the anti-HER2 CAR for its affinity near the top of the common range, and in order to avoid double-transfection (of a human TCR along with a CAR) which otherwise would create significant experimental inefficiencies. For all experiments using murine T cells, cells were maintained in RPMI (Gibco \#11875) supplemented with $10 \%$ fetal bovine serum (Benchmark), $100 \mathrm{U} / \mathrm{mL}$ penicillin, $0.1 \mathrm{mg} / \mathrm{mL}$ streptomycin, $2 \mathrm{mM}$ L-glutamine, 10 mM HEPES (Thermo \#15630106), $55 \mu \mathrm{M}$ beta-mercaptoethanol (Thermo \# 21985023), non-essential amino acids (Thermo \#11140050), 1mM sodium pyruvate (SigmaAldrich \#S8636), and supplemented with $100 \mathrm{U} / \mathrm{ml} \mathrm{IL-2,} \mathrm{which} \mathrm{is} \mathrm{referred} \mathrm{to} \mathrm{as} \mathrm{complete} \mathrm{RPMI.}$ 
Single cell suspensions were prepared from the lymph nodes and spleens of C57BL/6J, Ptprc ${ }^{a}$ (CD45.1), or OT-I TCR transgenic mice. Following red blood cell lysis of splenocytes, negative selection using the EasySep Mouse T Cell or CD8+ T cell Isolation Kit (STEMCELL Technologies \#19851 or \#19853) was used to isolate either all T cells (tumor growth experiments) or CD8+ T cells (in vitro experiments). T cells were activated in complete RPMI using CD3/CD28 Mouse T activator Dynabeads (Thermo \#11-453-D) for 24 hours before the first round of retroviral transduction.

For retrovirus production, Platinum-E cells were transfected with pMIG including CAR transgene using FuGene. Transfections were performed in DMEM (Gibco \#11995) supplemented with 10\% fetal bovine serum (Benchmark) and 10 mM HEPES (Thermo \#15630106), which was replaced with complete RPMI (without IL-2) the following day. Retroviral supernatants were harvested at day 2 and 3 and stored in $-80^{\circ} \mathrm{C}$. Platinum-E cells were maintained in DMEM (Gibco \#11995) supplemented with 10\% fetal bovine serum (Benchmark), $100 \mathrm{U} / \mathrm{mL}$ penicillin, $0.1 \mathrm{mg} / \mathrm{mL}$ streptomycin, $2 \mathrm{mM} \mathrm{L-glutamine} \mathrm{(Gibco} \mathrm{\# 10378),} 10$ mM HEPES (Thermo \#15630106), $10 \mu \mathrm{g} / \mathrm{ml}$ blasticidin (Fisher Scientific \#A1113903) and 1 $\mu \mathrm{g} / \mathrm{ml}$ puromycin (Gibco \#A1113803).

For T cell retroviral transduction, retroviral supernatant was added to T cells in retronectincoated plates at 24 and 48 hours following initial stimulation, and the plates were centrifuged for 1 hour at $2,000 \mathrm{~g}$ and $30^{\circ} \mathrm{C}$. After the second spinfection, cells were rested two days prior to Dynabead removal (4 days post-stim). T cells were then used for tumor growth experiments, sorted for imaging, or rested for an additional 6-7 days in $10 \mathrm{ng} / \mathrm{ml}$ recombinant murine IL-7 (PeproTech \#217-17) and $100 \mathrm{U} / \mathrm{ml} \mathrm{IL-2} \mathrm{and} \mathrm{used} \mathrm{for} \mathrm{in} \mathrm{vitro} \mathrm{co-culture} \mathrm{experiments.}$ 
For binned HER2 expression level experiments, MC38-HER2 lines were sorted into 5 consecutive bins by HER2 expression using MCF7s and SKBR3s as the low and high HER2 standards, respectively. MC38-HER2 cells, sorted as described, were then plated at $5 \times 10^{4}$ cells/well in flat-bottom 96 well plates, and $5 \times 10^{4} \mathrm{~T}$ cells were then added, bringing the total volume to $200 \mu \mathrm{l} /$ well complete RPMI. For hypoxia experiments, T cells were rested 6 days and then plated at $5 \times 10^{4}$ cells at a 1:1 ratio with MC38-HER2-high or MC38-HER2-low cells in two 96-well plates in complete RPMI. At 24 hours, wells were replenished with complete RPMI + IL2, and $5 \times 10^{4}$ MC38-HER2-high and -low cells were added. One plate was moved to $1.5 \%$ oxygen while the second was maintained at $20 \%$ oxygen. MC38-HER2-high and -low cells and media + IL-2 were then replenished every two days until analysis on day 7 after start of coculture (6 days in hypoxia).

\section{In vivo CAR T cell tumor experiments}

Sex- and age-matched mice of age 8-12 weeks were used for all tumor experiments. $4 \times 10^{5}$ MC38-HER2-high or MC38-HER2-low cells were injected subcutaneously in $50 \mu \mathrm{l}$ of 1:1 PBS:Matrigel (Growth Factor Reduced Basement Membrane Matrix, Phenol Red-free Corning \# 356238). Mice were then treated with sub-lethal $5 \mathrm{~Gy}$ irradiation the following day, and $3 \times 10^{6}$ CAR (or untransduced) T cells were retro-orbitally transferred in PBS. T cells were transduced as described above and numbers transferred were adjusted according to percent CARmEmerald+ as defined by flow cytometry. Tumors were measured by caliper and harvested for flow cytometric analysis at day 21-24. At harvest, tumors were finely minced and then digested with $500 \mathrm{U} / \mathrm{ml}$ Collagenase IV (Worthington Biochemical \#LS004189), 100 U/ml Collagenase I (Worthington Biochemical LS004197), and 200 mg/ml DNAse I (Sigma-Aldrich \#10104159001) in RPMI with shaking for 30 min at $37^{\circ} \mathrm{C}$, with vortexing every ten min. Cells were then washed in PBS with $2 \%$ FCS, passed through a $100 \mu \mathrm{m}$ filter, and counted prior to staining for flow cytometry. 


\section{Surface plasmon resonance affinity measurements}

Measurements were taken using a Biacore T200 instrument with CM4 sensor chip and HBsEP+ buffer. HER2-mlgG2aFc (ACROBiosystems \#HE2-H5255) was captured using anti-mlgG (50 RUs). Association and dissociation times were 120 and $900 \mathrm{sec}$, respectively. Concentrations of scFv used for single cycle kinetics analysis: 0.33, 1, 3, 9, $27 \mathrm{nM}$.

\section{Lattice Light-Sheet Microscopy}

Lattice light-sheet imaging was performed as described previously ${ }^{8} .5 \mathrm{~mm}$ round coverslips were cleaned by a plasma cleaner, and coated with $2 \mu \mathrm{g} / \mathrm{ml}$ fibronectin in PBS at $37^{\circ} \mathrm{C}$ for 1 hour, or at $4^{\circ} \mathrm{C}$ overnight, before use. $\sim 3 \times 10^{5} \mathrm{CAR} T$ cells were loaded onto the coverslip and incubated at $37^{\circ} \mathrm{C}$ for $30 \mathrm{~min}$. Cells were then fixed in $\mathrm{d}_{2} \mathrm{~h}_{2} \mathrm{O}$ with $20 \mathrm{mM}$ HEPES (Thermo \#15630106), 0.2 M sucrose (RPI \#S240600, 4\% paraformaldehyde (Election Microscopy Sciences \#15710), and 8\% glut-aldehyde (Election Microscopy Sciences \#16019) for $10 \mathrm{~min}$ at room temperature. Coverslip was washed gently in $1 \mathrm{ml} \mathrm{PBS}$ and then stained with antibodies to CD45 and/or MYC with anti-mouse Fc receptor antibody (clone 2.4G2, UCSF Hybridoma Core). Samples were stained for at least $30 \mathrm{~min}$ and kept at $4^{\circ} \mathrm{C}$ until use. Prior to imaging, coverslip was gently washed with $1 \mathrm{ml}$ warmed RPMI without phenol red (Gibco \#11835) supplemented with $2 \%$ fetal bovine serum, $100 \mathrm{U} / \mathrm{mL}$ penicillin, $0.1 \mathrm{mg} / \mathrm{mL}$ streptomycin, $2 \mathrm{mM}$ L-glutamine, $10 \mathrm{mM}$ HEPES and $50 \mu \mathrm{M} \beta$-mercaptoethanol (imaging media). For imaging of live CAR T cell interactions with MCF7 and SKBR3 targets, MCF7 or SKBR3 cells were plated onto fibronectin-coated coverslips 1-2 days prior to imaging. CAR T cells were stained with antibody to CD45-Alexa647 for 30 min on ice. Target cells on coverslip were stained with CFSE (Invitrogen \#C34554) for $20 \mathrm{~min}$ at $37^{\circ} \mathrm{C}$. Cells were then washed and T cells were added onto the coverslip prior to being loaded into the sample bath with warmed imaging media and secured. Imaging was performed with a $488 \mathrm{~nm}, 560 \mathrm{~nm}$, or $642 \mathrm{~nm}$ laser (MPBC, Canada) 
dependent upon sample labeling. Exposure time was $10 \mathrm{~ms}$ per frame leading to a temporal resolution of $\sim 4.5$ and $\sim 6.75$ seconds in two- and three-color mode respectively.

\section{Supported lipid bilayers, synaptic contact mapping, and calcium flux imaging}

Preparation and use of supported lipid bilayers was performed as described previously ${ }^{8,45}$. Mixtures of $96.5 \%$ POPC, $2 \%$ DGS-NTA (Ni), 1\% Biotinyl-Cap-PE and 0.5\% PEG5,000-PE (Avanti Polar Lipids \#850457C, 790404C, 870273C, 880230C) were made in a round bottom flask and dried under a stream of nitrogen and then overnight under vacuum. The phospholipids were then rehydrated at a total concentration of $4 \mathrm{mM}$ in PBS for one hour to create crude liposomes. Small, unilamellar liposomes were then made by extruding through $100 \mathrm{~nm}$ Track Etch filter papers (Whatman \#800309) with an Avestin LiposoFast Extruder (Avestin).

8-well Nunc Lab-Tek II chambered coverglass (Thermo \#155360) were cleaned by submersion in 5\% Hellmanex III (Sigma-Aldrich \#Z805939). Flask containing chamber in solution was microwaved for $25 \mathrm{sec}$ and then allowed to clean at room temperature overnight. The chambers were then washed repeatedly with 18 Milli-Q water and then dried. Finally, $250 \mu \mathrm{l} 3 \mathrm{M} \mathrm{NaOH}$ was added to each well for $15 \mathrm{~min}$ at $55^{\circ} \mathrm{C}$. Wells were washed with $300 \mu \mathrm{l}$ Milli-Q water and the $\mathrm{NaOH}$ cleaning was repeated. Wells were then washed thoroughly and dried prior to use. Lipid bilayers were set up on the chambered coverglass by adding $0.25 \mathrm{~mL}$ of a $0.4 \mathrm{mM}$ liposome solution to the wells. After 30 minutes, wells were rinsed with $8 \mathrm{~mL}$ of PBS by repeated addition of $0.5 \mathrm{~mL}$ of PBS, then aspiration of $0.5 \mathrm{~mL}$ of the overlay. Non-specific binding sites were then blocked with 1\% BSA in PBS for 30 minutes. After blocking, 25 ng of unlabeled streptavidin (Invitrogen \#43-4301) was added to each well and allowed to bind to bilayers for 30 minutes. After rinsing, protein mixes containing 63 ng recombinant human ICAM-1 (Abcam \#AB151393) and 6.25-625 ng biotinylated HER2 (ACROBiosystems \#HE2-H822R) or 6 ng pMHC in 2\% BSA were injected into each well. pMHC was provided by the NIH Tetramer Facility. After binding for 
30 minutes, wells were rinsed again and $25 \mathrm{ng}$ of QDot605-streptavidin (Thermo \#Q10101MP) was added to each well. For calcium flux imaging, QDot605-streptavidin was not added. Bilayers were finally rinsed with imaging media before being heated to $37^{\circ} \mathrm{C}$ for experiments. Experiments using $5 \mu \mathrm{m}$ diameter silica microspheres (Bangs Laboratories \#SS05003/SS05N) were performed as previously described ${ }^{46}$. Briefly, the same protocol was used for building a lipid bilayer on chamber coverglass as for $4 \times 10^{5}$ beads, based off equivalent surface area, but with washes performed by centrifugation instead of repeated aspiration.

For synaptic contact mapping (SCM) experiments, $5 \times 10^{5} \mathrm{~T}$ cells were added to the well prior to imaging. Once cells began interacting with the bilayer, imaging was initiated. For imaging of OTI TCR, 1-2×106 OT-I T cells were stained with $2.5 \mu$ g H57-597 non-blocking monoclonal antibody conjugated to Alexa Fluor 488 on ice for 30 minutes, then rinsed once with complete imaging media. Imaging for synaptic contact mapping was performed as described previously ${ }^{8}$. The TIRF microscope is based on a Zeiss Axiovert 200M equipped with a 100x 1.45NA oil immersion objective, DG-4 Xenon light source (Sutter) and Zeiss TIRF slider ${ }^{8,45}$. All images were collected using a DV2 image splitter (Photometrics) positioned in front of an Evolve EMCCD (Photometrics). A 4 band multi-color TIRF dichroic located in the microscope separated the excitation and emission light for imaging (Chroma Technology). Images of CAR or TCR were collected by imaging CAR-mEmerald (or Alexa Fluor 488-labeled TCRs) using TIRF mode, by imaging QD605-streptavidin in widefield mode, and by imaging cells with interference reflection microscopy (IRM), also in widefield mode. Widefield QD605-strepavidin images were collected using a 405/10 nm excitation filter (Chroma Technology) located in the DG4 light source, while samples imaged with TIRF were excited by an Obis 488nm laser (Coherent). IRM images were acquired using a 635/20 nm excitation filter (Chroma Technology) positioned in the DG4 light source. The CAR/TCR and QD605 emitted fluorescence signals were separated using a DV2 image splitter with a $565 \mathrm{~nm}$ long-pass dichroic mirror installed along with 520/35 
$\mathrm{nm}$ and 605/70 nm emission filters (Chroma). Images containing the IRM signal were acquired through the long-pass dichroic and 605/70 nm emission filter in the image splitter. For Fura-2 imaging, cells were stained with $2 \mu \mathrm{M}$ Fura-2 dye (Thermo \#F1221) for $15 \mathrm{~min}$ at room temperature. Cells were then washed in imaging media, and $5 \times 10^{5} \mathrm{~T}$ cells were added to the imaging well. 3 min after addition of cells, acquisition was initiated. Fura-2 imaging experiments were acquired using a 40x 1.3NA oil immersion objective (Zeiss) and the same light source and dichroic described above. Widefield Fura-2 $340 \mathrm{~nm}$ and $380 \mathrm{~nm}$ images were collected using 340/26 (Semrock, Rochester, NY) and 380/30 (Chroma, VT) excitation filters, respectively, located in the DG4 light source.

\section{Image analysis}

All computational image analysis for SCM imaging was performed in Matlab (The Mathworks, Natick, MA), Imaris version 9.2.1 or 7.6.3 (Bitplane), and Fiji. CAR-mEmerald microcluster tracking analysis was performed using the spots function in Imaris with the following parameters: $0.25 \mu \mathrm{m}$ estimated diameter, autoregressive motion tracking, $0.5 \mu \mathrm{m}$ maximum distance, gap size 3 , track duration $>5 \mathrm{sec}$. Centroid positions for dot product calculations were defined by making a surface of the synapse CAR-mEmerald interface in Imaris with grain size of $3 \mu \mathrm{m}$ and largest sphere diameter of $1 \mu \mathrm{m}$. Analysis for LLS was performed in Imaris and Matlab. Unique analysis code has been made available through GitHub and can be found at the URLs included below.

\section{Lattice Light-Sheet: Post Processing}

Raw data were deconvolved using the iterative Richardson-Lucy deconvolution process with a known point spread function that was recorded for each color prior to the experiment, as described previously ${ }^{8}$. A typical sample area underwent 15-20 iterations of deconvolution. For 
live imaging experiments, photobleaching correction was applied in FIJI using the histogram matching method.

\section{Close contact segmentation and persistence analysis}

Close contact segmentation, CAR co-localization, and persistence time analysis was performed using Matlab and Imaris as previously described ${ }^{8}$. Briefly, the IRM images were used to define the region of the cell interface. Active contour segmentation of the QD605 image was then used to define close contact regions. These regions were then converted to Spots objects in Imaris. CAR intensity was masked to regions of close contacts, and the average intensity for each contact area was then plotted in a histogram. A Gaussian distribution curve centered at the background fluorescence median was overlayed. Contacts that fell within 3 sigma of the Gaussian distribution were considered $\mathrm{CAR}^{-}$, while the higher intensity contacts were considered $\mathrm{CAR}^{+}$. These contacts were then separated into separate image stacks and persistence time for individual contacts were calculated. Contact persistence time was determined by summing the number of frames each binary connected component object existed for and multiplying by the time per frame. Contacts were assumed to not travel more than their diameter per time point. Code for analysis of persistence times has been made available: https://github.com/BIDCatUCSF/NanocontactsTIRF V5.

\section{Radial Intensity Profiles}

Definition of cell boundary, assignment of radial coordinates, and plotting of pixel intensities were performed using MatLab (Natick, MA). The outer edge (boundary) of cells was detected using a custom program which primarily applied a two-step kmeans clustering calculation on each of the image slices collected in the z-stack of images describing a single cell. The boundary was then eroded by three pixels to accommodate the resolution of the LLS imaging 
system. Code for defining the cell boundary: https://github.com/BIDCatUCSF/Exterior-t-CellEdge-Detection. Radial coordinates were assigned for plotting of radial intensity profiles using this code: https://github.com/BIDCatUCSF/Outer-Boundary-Profile-Code. Binned intensity was measured by taking a moving average of ten pixels. Excursions of the binned intensity above the channel mean intensity +3 s.d. were used as the threshold to define patches.

\section{Fura-2 ratio image analysis}

Fura-2 340nm/380nm ratio images were created in MetaMorph Version 7.6.5.0 using a maximum ratio of 7 and imported to Imaris for tracking using the surface function with the following parameters: $1 \mu \mathrm{m}$ grain size, $0.75 \mu \mathrm{m}$ diameter of largest sphere, $2 \mu \mathrm{m}$ region growing estimated diameter autoregressive motion tracking, $2 \mu \mathrm{m}$ maximum distance, gap size 1, track duration above $148 \mathrm{sec}$. For analysis of final time point, surfaces were made for all cells in field of view without tracking: $0.75 \mu \mathrm{m}$ grain size, $0.75 \mu \mathrm{m}$ diameter of largest sphere, $1.5 \mu \mathrm{m}$ region growing estimated diameter. Maximum 340nm/380nm ratio per cell was compared for all cells in field of view at final imaging time point -5.5 min after addition of $\mathrm{T}$ cells to chamber well.

\section{Statistics}

Statistical tests were performed using GraphPad Prism Version 9.0.1. Independent experiments and donors are as noted in figure legends - all other replicates are technical replicates. All $t$ tests performed were two-sided. Scatter plots show mean and s.d (error bars). Box and whisker plots indicate median, $25^{\text {th }}$ to $75^{\text {th }}$ percentile (box), and minimum to maximum (whiskers). $\mathrm{P}$ values are reported as follows: $\geq 0.05$ as ns, 0.01 to 0.05 as ${ }^{*}, 0.001$ to 0.01 as ${ }^{* *}, 0.0001$ to 0.001 as $^{* * *}$, and $<0.0001$ as $^{* * * *}$. 
A

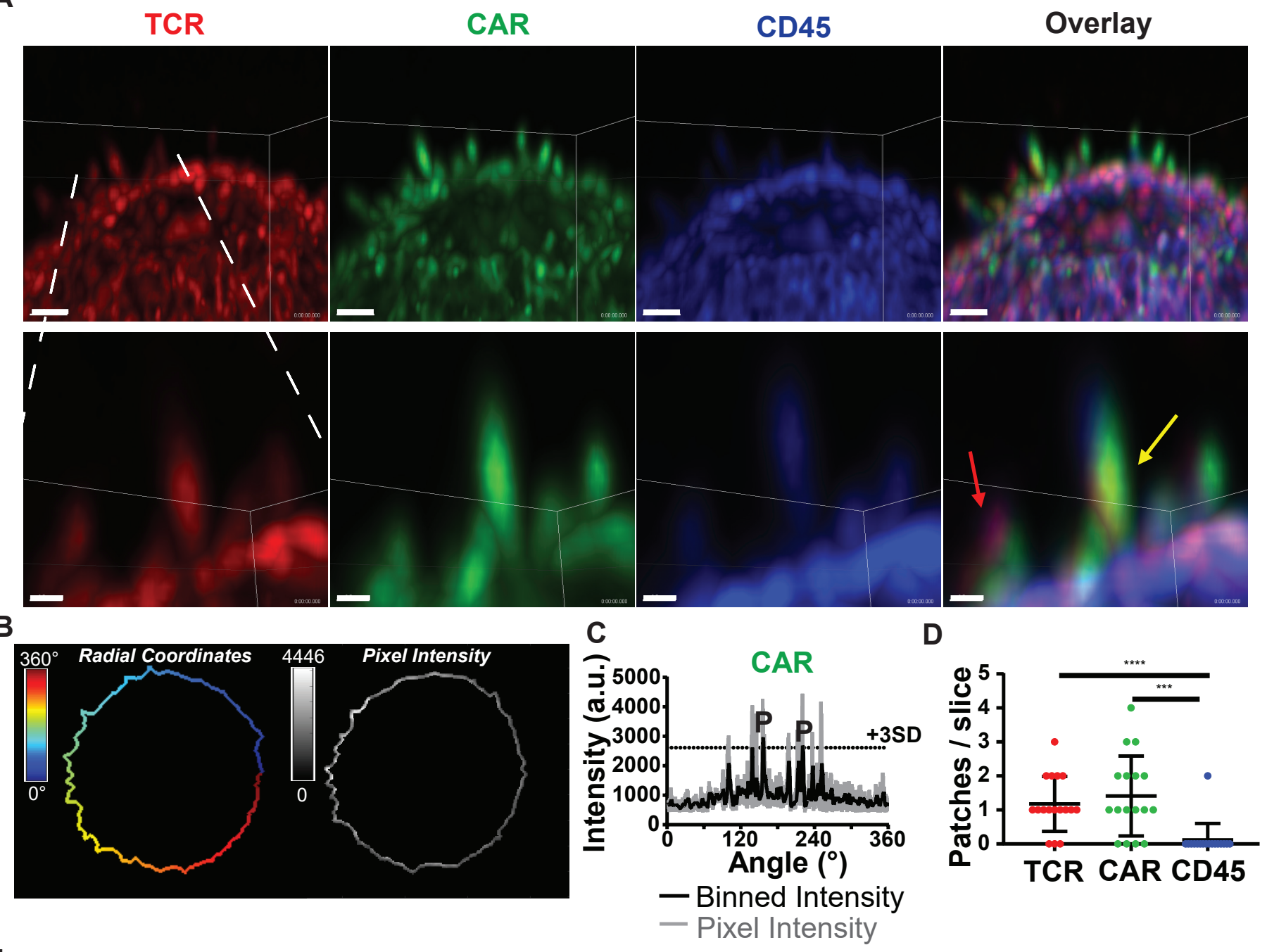

E

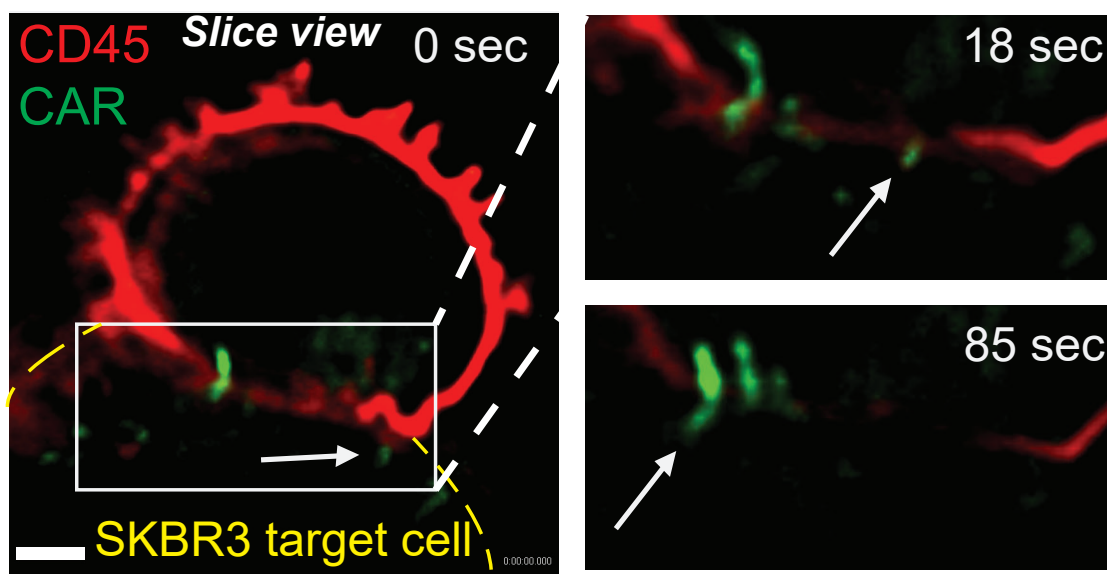

F

CAR

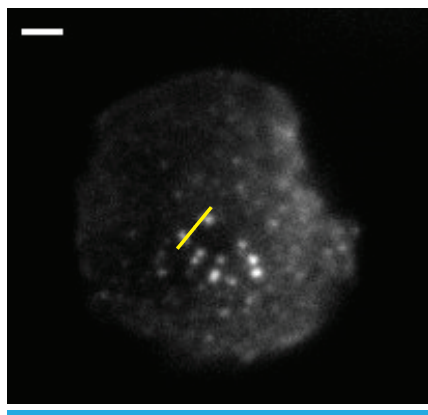

Lipid bilayer loaded with HER2 + ICAM + QD605
QD605

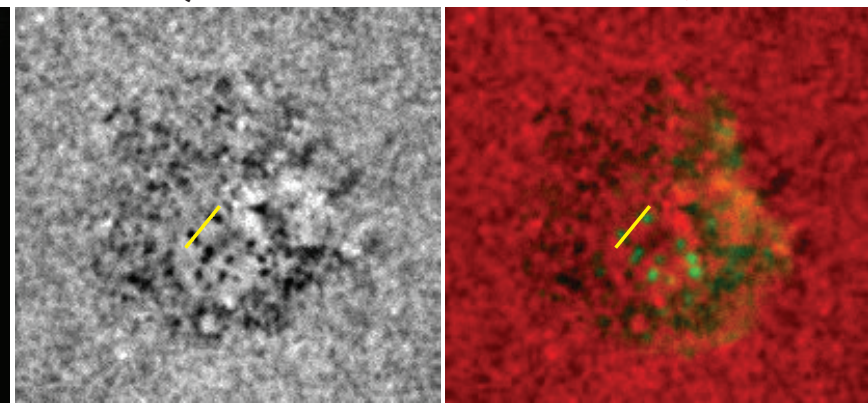

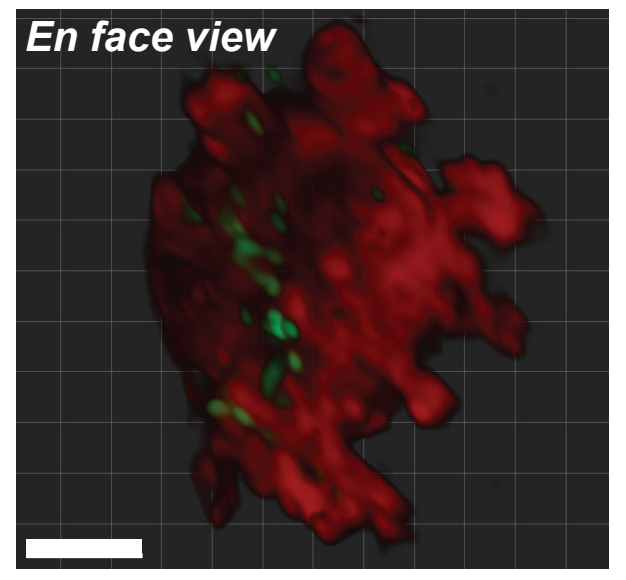

G

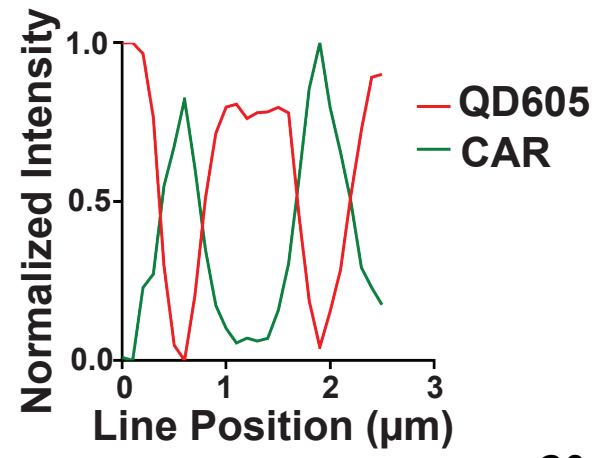


Figure 1: CARs distribute as patches, which localize to projections into the synapse following interaction with a stimulatory surface.

A. Fixed anti-HER2 CAR T cell showing maximum intensity projection of CAR, TCR, CD45 and overlay imaged by lattice light-sheet. Bottom row shows zoomed view of region with one microvillus occupied by TCR but not CAR (red arrow), and one occupied by both CAR and TCR (yellow arrow). Top scale bar $=2 \mu \mathrm{m}$. Bottom scale bar $=0.7 \mu \mathrm{m}$. Human CD8+ T cell expressing anti-HER2 CAR (mut4D5) was labelled with antibodies to MYC (CAR)-Alexa488, TCR (OKT3)-APC, and CD45-Alexa594.

B. Schematic showing the cell boundary as mapped to radial intensity profile by angle (left) and pixel intensity (right).

C. Pixel intensity (gray line) and binned intensity (black line), defined by taking a moving average of 10 pixels, here showing one z-slice for CAR-mEmerald. Dashed line indicates 3 standard deviations (+3SD) above mean pixel intensity. $P$ indicates binned intensity peaks above +3SD threshold (patches).

D. Number of patches/z-slice for each receptor was defined by the number of excursions of binned intensity above the $+3 S D$ threshold. $T$ test was performed on data compiled from multiple slices of 3 cells ( $n=17$ slices per group). Error bar represents standard deviation (s.d.).

E. Anti-HER2 CAR T cell interacting with target HER2+ SKBR3 cell (left: side view slice with SKBR3 location marked by dashed yellow line, scale bar $=2 \mu \mathrm{m}$; center: zoomed on white box region at 18, 85 seconds later; right: en face synapse surface, scale bar $=4 \mu \mathrm{m}$ ), showing CAR enrichment at projections into the synapse (white arrows). Human CD8+ T cell expressing antiHER2 CAR (4D5) was labelled with antibodies to MYC (CAR)-Alexa488 and CD45-Alexa647.

F. CAR T cell synapse imaged by TIRF showing CAR-mEmerald, microvillar projections (as seen by holes in QD605 signal), and overlay. Human CD8+ T cell expressing anti-HER2 CAR (mut4D5) interacting with lipid bilayer loaded with 625ng HER2 + ICAM + QD605. Scale bar is $2 \mu \mathrm{m}$.

G. Line scan from $F$ shows anti-correlation of CAR-mEmerald and QD605, indicating enrichment of CARs within microvillar contacts. 
A

Low affinity
scFv
$K_{D}: 3.63 n M$
High affinity
scFv
$K_{D}: 0.0523 n M$

C

Microvillar Close Contacts

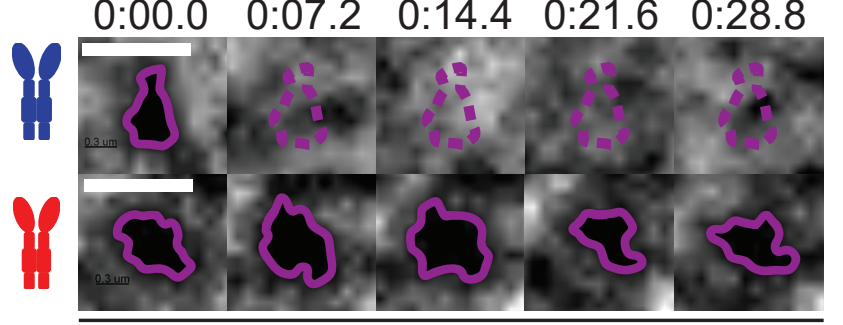

HER2 (ng/well): 6.25
B

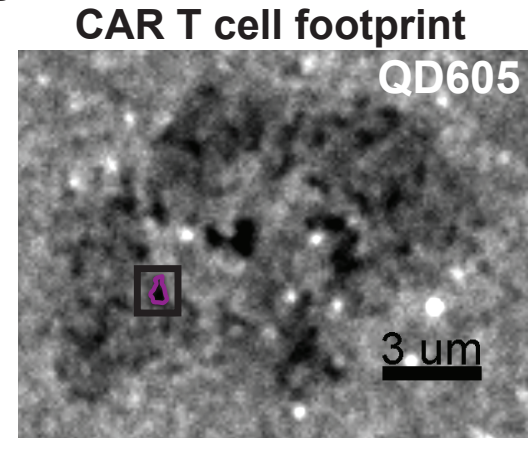

D

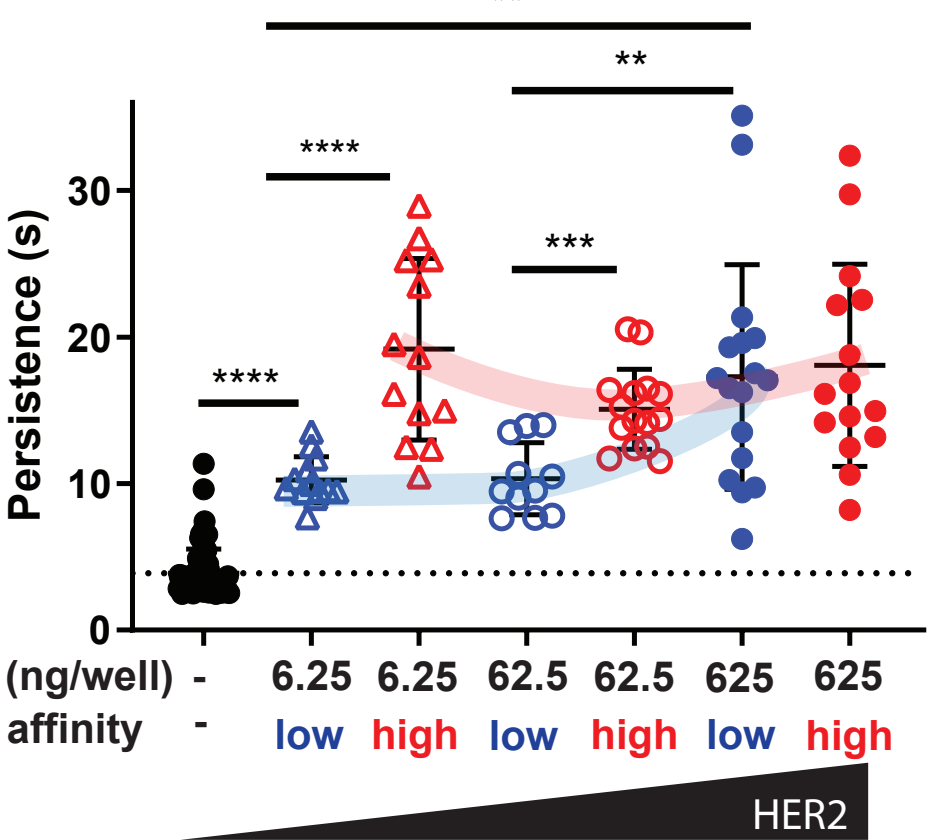

E

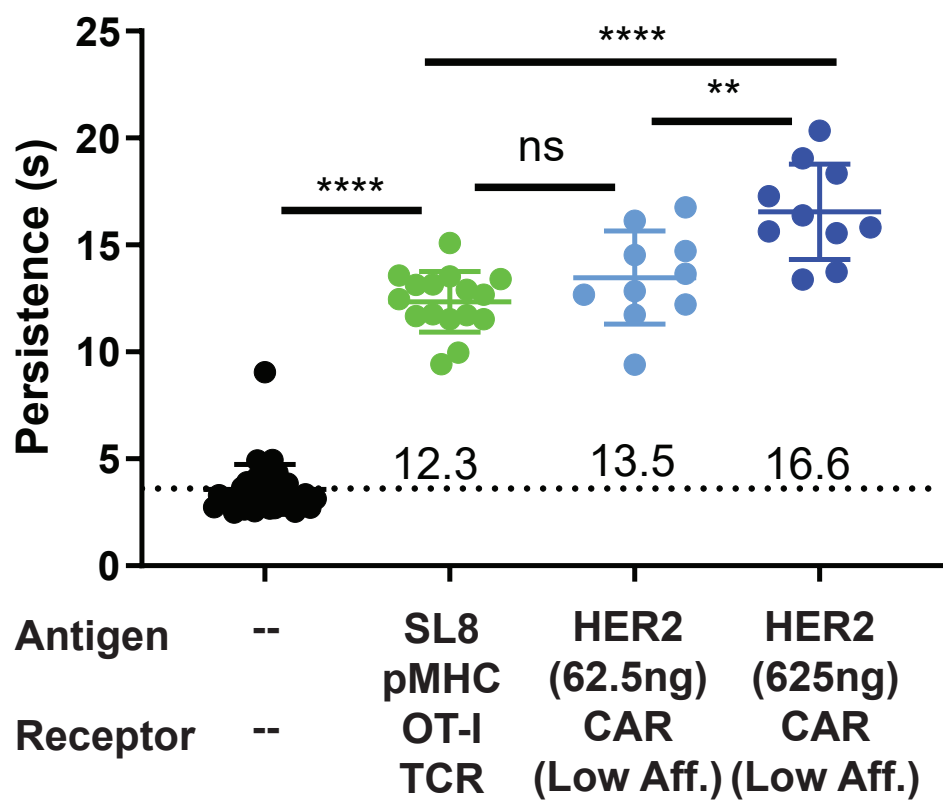

$\mathbf{F}$

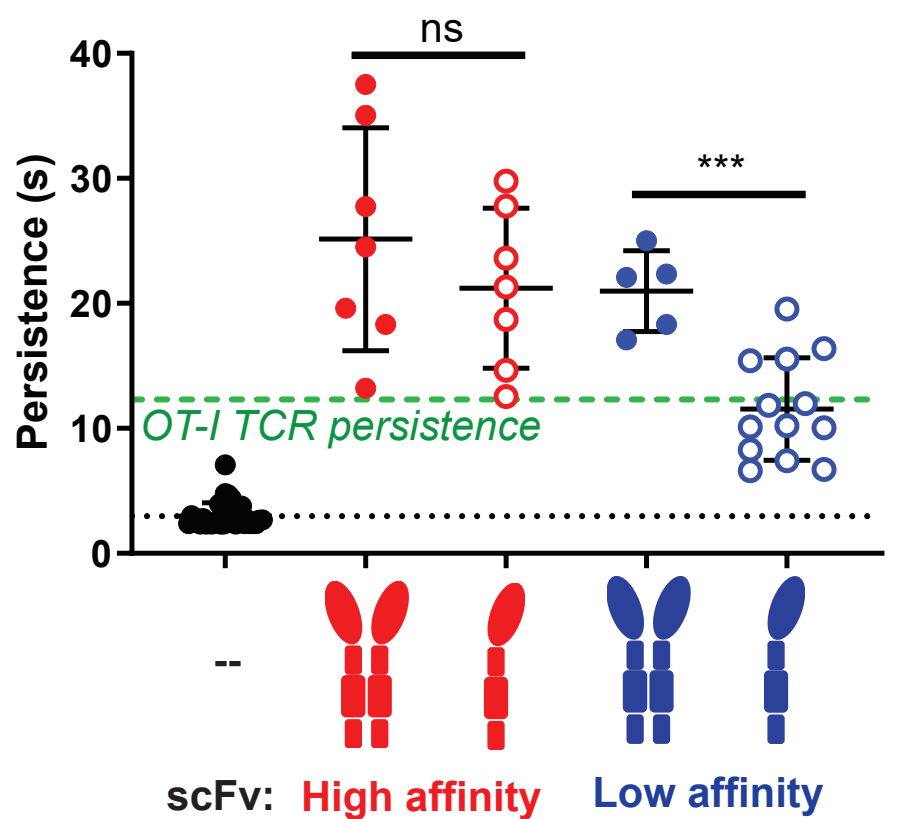


Figure 2: Conventional CAR interactions of high affinity or high antigen density result in hyper-stabilization of underlying microvillar protrusion, which can be reduced by using monomeric CAR.

A. Experiments were performed comparing CARs with a high-affinity scFv against HER2-based off trastuzumab (4D5, $\mathrm{K}_{\mathrm{D}}=0.0523 \mathrm{nM}$, red) and a lower affinity scFv made by substitution of three amino acids (see Supplemental Table 1, mut4D5, $\mathrm{K}_{\mathrm{D}}=3.63 \mathrm{nM}$, blue).

B. Bilayers were loaded with fluorescent quantum dots (QD605) with a height of 16nm.

Locations where the cell makes close contact with the bilayer $(<16 \mathrm{~nm})$ are visualized as holes in QD605 signal due to their size-based exclusion. QD605 signal is shown for a low-affinity CAR T cell interacting with a lipid bilayer loaded with 6.25 ng HER2. Outlined contact is shown in C.

Scale bar is $3 \mu \mathrm{m}$.

C. QD605 signal across 5 time points are shown for the same field of view, each on low HER2 bilayers (6.25 ng/well). Top: Microvillus from low-affinity CAR T cell moves out of view. Bottom: Microvillus from high-affinity CAR T cell remains across time points. White scale bar $=1 \mu \mathrm{m}$.

D. CAR-occupied close contact persistence times (blue, red) and CAR-negative close contact persistence times (black) are shown. All CAR:HER2 interactions tested result in CAR-occupied microvillar contact stabilization above background CAR-negative contacts (black, dotted line). Persistence time is further increased in interactions of high-affinity CAR (red), even at lowest HER2 densities on the bilayer. For low-affinity CAR (blue), only high levels of HER2 yield similar persistence times to high-affinity CAR. Data is shown for at least 11 cells per condition across four experiments ( $n=84,13,13,11,15,17,15$ cells per group from left to right, respectively).

E. Low-affinity CAR was retrovirally expressed in primary mouse OT-I CD8+ T cells. Receptoroccupied microvillar persistence times are shown for OT-I:SL8 (green), Low-affinity CAR:Low HER2 (light blue), and Low-affinity CAR:High HER2 (dark blue) interactions. All cognate interactions are stabilized above background receptor-negative contacts (black, dotted line). CAR:High HER2 persistence is hyper-stable relative to TCR:pMHC stabilization. Data is shown for at least 10 cells per condition across three experiments $(n=36,16,10,10$ cells per group from left to right, respectively).

F. Dimers (filled dots) and monomers (open dots) are compared on high HER2 bilayers (625ng/well). Only monomeric low-affinity CAR regains natural persistence time of TCR:pMHC contacts (green dashed line). All receptor-occupied contacts are stabilized above non-cognate antigen interactions (black, dotted line). Data is shown for at least 5 cells per condition across three experiments ( $n=32,7,7,5,13$ cells per group from left to right, respectively). All error bars represent s.d. and analyses shown are unpaired t tests. 
A

\subsection{5ng HER2}

625ng HER2

C

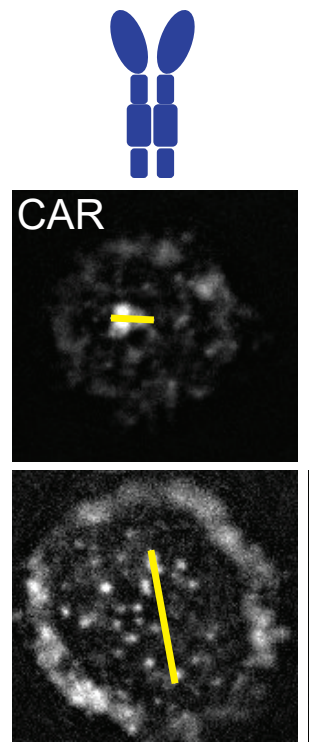
available under aCC-BY-NC-ND 4.0 International license.

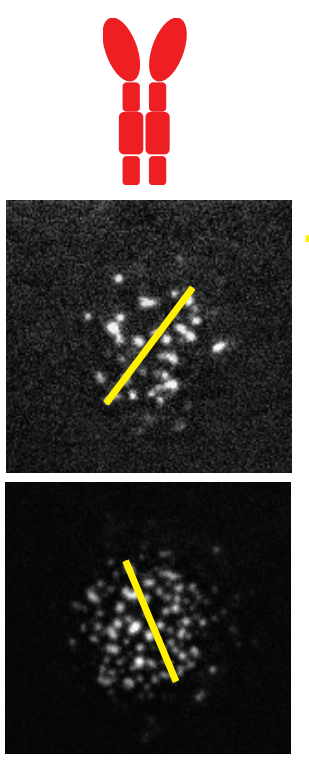

B

HER2 (ng/well) $6.25 \quad 6.25 \quad 625 \quad 625 \quad 625 \quad 625$ scFv affinity low high low high low high Dimer Monomer

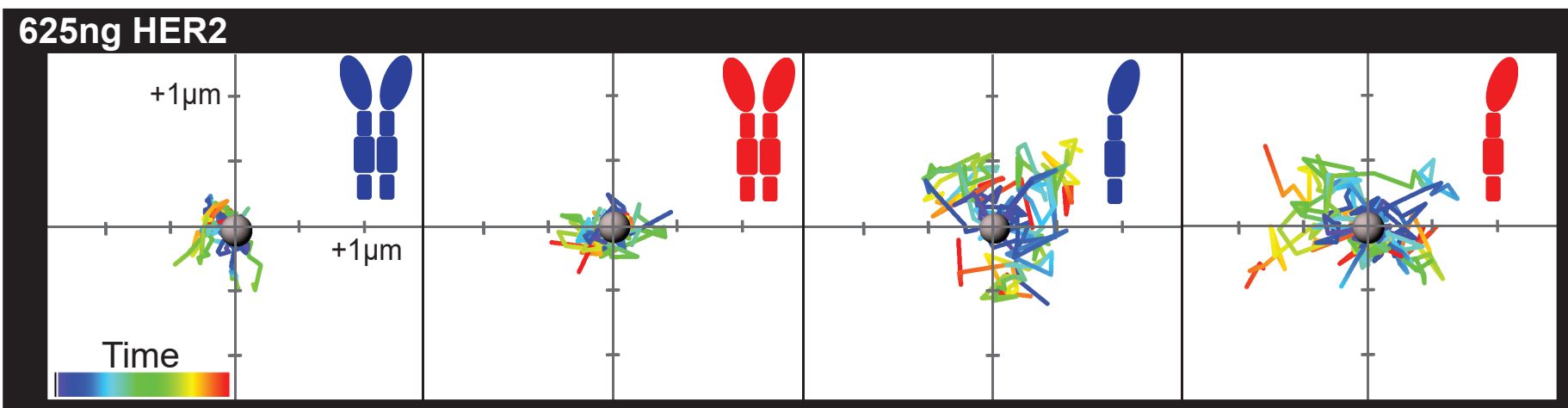

\subsection{5ng HER2}

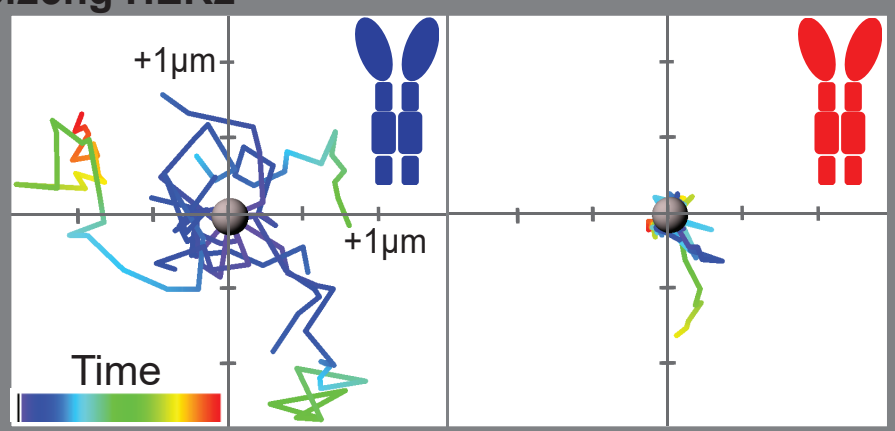

D

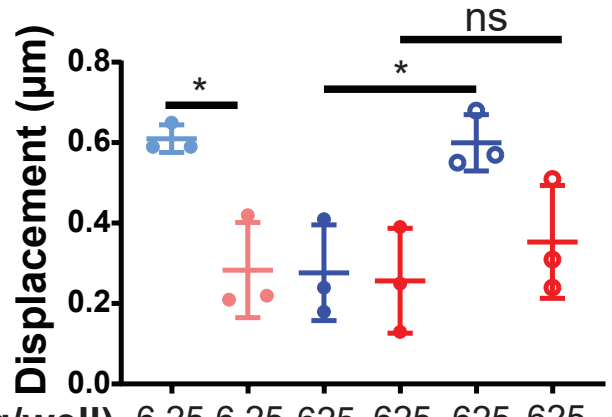

HER2 (ng/well) 6.256 .25625625625625

scFv affinity low high low high low high 
Figure 3: Impaired movement of CAR microclusters in synapses.

A. TIRF imaging CAR-mEmerald is shown for synapses with low- or high-affinity CAR interacting with low or high HER2-loaded bilayers. Time point shown is 93.6 seconds following the initiation of imaging, which began as synapses were starting to form. Yellow line spans CAR microclusters in synapse, indicating diameter quantified in B.

B. Diameter across CAR microclusters in synapse (yellow lines in A). For dimeric CARs, only low affinity:low HER2 synapses result in accumulation of CAR microclusters at the center, indicated by lower diameter. Monomerization of CARs improves centralization on high HER2, especially in the low-affinity CAR. Data is shown for at least 7 cells pooled from a minimum two independent experiments per condition $(n=13,13,20,20,13,7$ cells per group from left to right, respectively).

C. Spots with tracking were assigned to CAR microclusters in Imaris, and a random 10 tracks are shown in flower plots for each condition. Top: Limited mobility is apparent for dimeric CARs on high-HER2 loaded bilayers. Bottom: On low-HER2 bilayers, low-affinity CAR microclusters show increased mobility.

D. Quantification of the average displacement of all CAR microclusters for a given cell $(n=3$ cells per group). All error bars represent s.d. and analyses shown are unpaired t tests. 


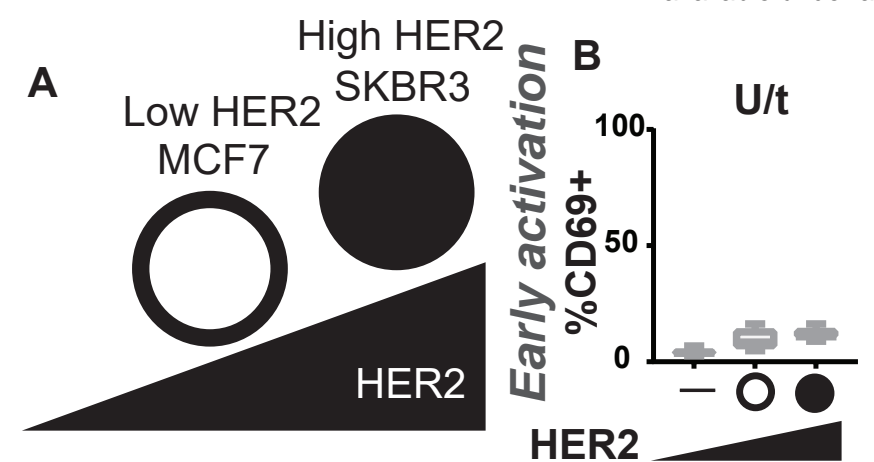

HER2

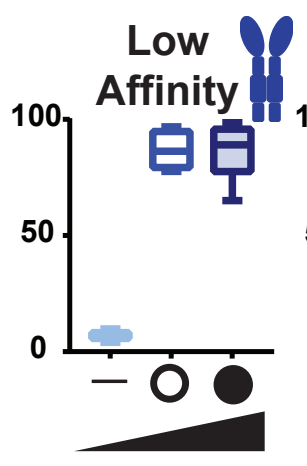

D

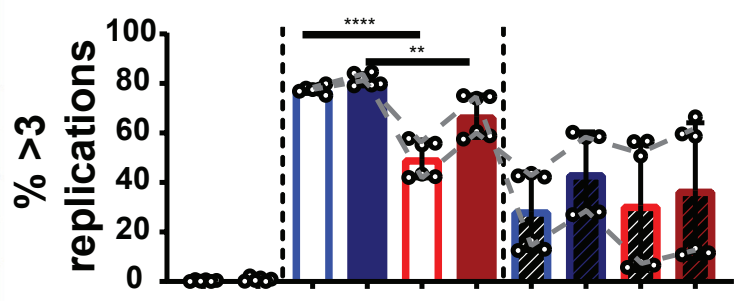

HER2: 0000000000

CAR:
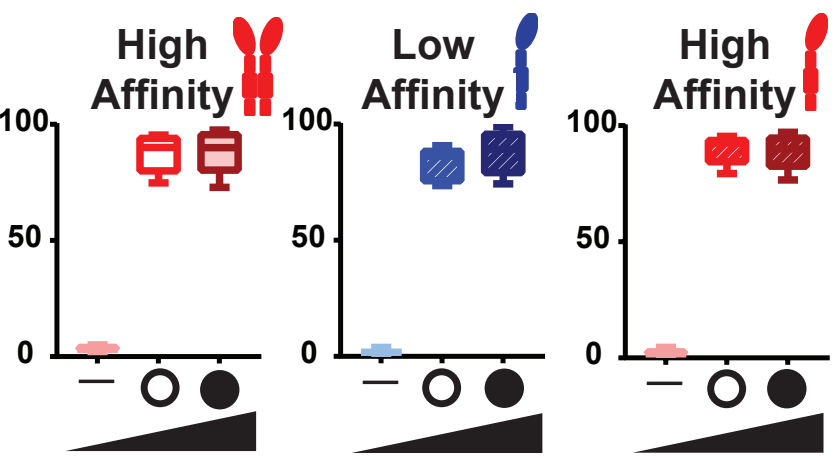

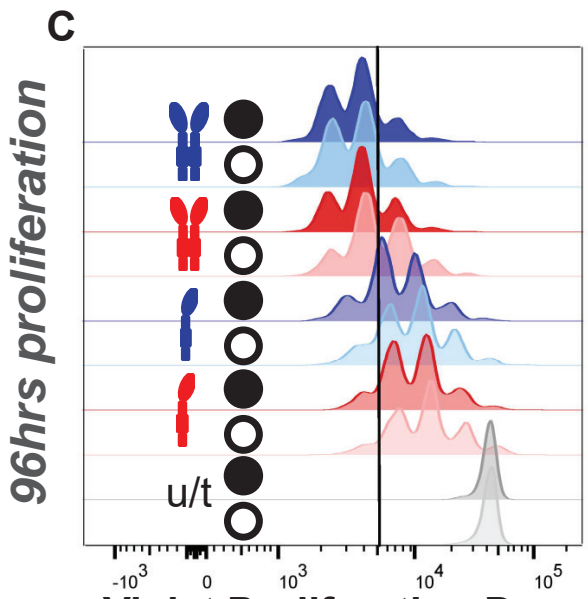

Violet Proliferation Dye
E

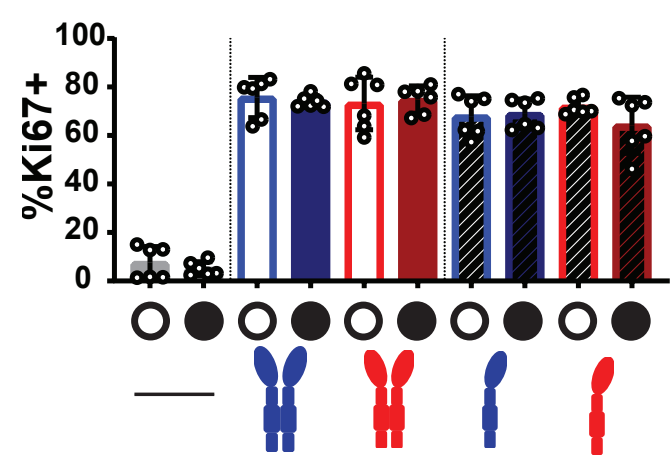

G

Hypoxia Co-Cultures

을
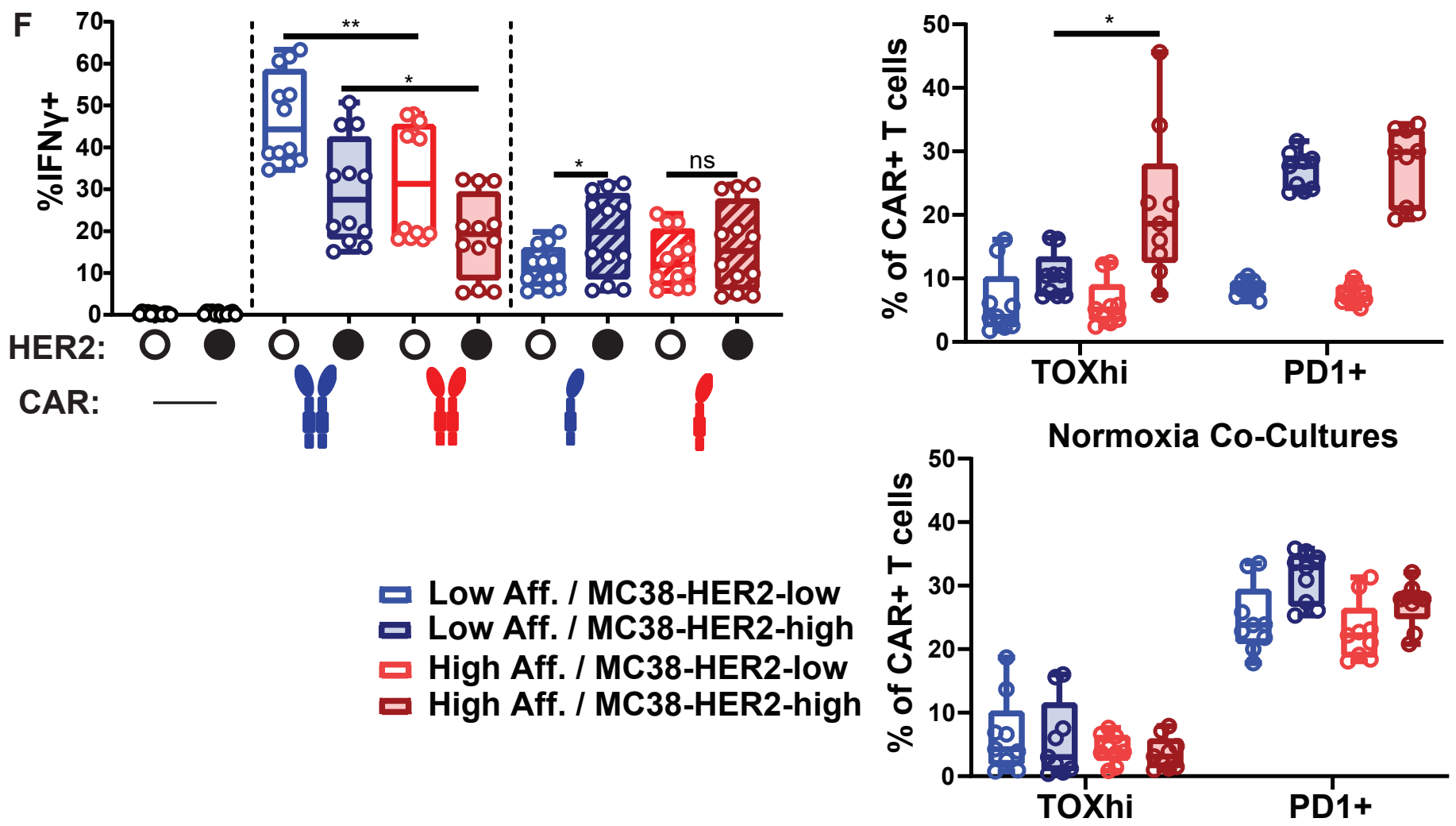
Figure 4: High-affinity CARs do not have advantage over lower affinity CARs, and both high and low-affinity dimers show insensitivity to antigen sensitivity.

A. Low HER2-expressing MCF7 (open circle) and high HER2-expressing SKBR3 (solid circle) cell lines were used to assess differences between HER2 levels in cell-cell interactions in vitro.

B. Early activation is similar across all CAR+ conditions with low and high HER2 at 18 hours following co-incubation, as seen by anti-CD69-BUV395 positivity. Box and whiskers plot error bars showing minimum and maximum values $(n=12$ samples per group pooled from four independent experiments and two donors).

C. Proliferation is induced across all CAR+ conditions with low and high HER2, as seen by VPD dilution at 96 hours following co-incubation. Line marks VPD dilution indicating at least 3 replication cycles (quantified in D).

D. Percentage of cells that have undergone at least 3 replication cycles. Replicates from 2 independent experiments of different donors are pooled $(n=6)$. Error bars represent s.d. Dashed lines indicate individual donor trends.

E. Percentage of anti-Ki67-PE-eFluor 610 positive cells is similar across all CAR+ conditions with low and high HER2. Replicates from 2 independent experiments of different donors are pooled $(n=6)$. Error bars represent s.d.

F. Intracellular staining with anti-IFN-Y-APC at 18hrs following co-incubation. Low-affinity CAR induces greater IFN- $y$ than high-affinity, and only monomeric low-affinity CAR, which has overall lower magnitude of response, shows sensitivity to antigen density. Box and whiskers plot error bars showing minimum and maximum values $(n=12$ samples per group pooled from four independent experiments and two donors).

G. T cells were co-cultured with MC38s expressing low- or high-HER2 for 24 hours in normoxia ( $20 \%$ oxygen) and then separated into normoxic or hypoxic $(1.5 \%$ oxygen) cultures for an additional six days. Under hypoxic conditions, high-affinity CAR interactions with high HER2 upregulate TOX, while PD-1 expression is upregulated in high HER2 interactions for both high and low-affinity CARs. Box and whiskers plot error bars showing minimum and maximum values ( $n=9$ samples pooled from 3 independent experiments). All analyses shown are unpaired $t$ tests. 